\title{
DESIGN OF ROBUST GUARANTEED COST PID CONTROLLER FOR NETWORKED CONTROL SYSTEMS
}

\author{
Quang Thuan Nguyen — Vojtech Veselý *
}

\begin{abstract}
The paper addresses the problem of an output feedback guaranteed cost controller design for Networked Control Systems (NCSs) with time-delay and polytopic uncertainties. By constructing a new parameter-dependent Lyapunov functional and applying the free-weighting matrices technique, the parameter-dependent, delay-dependent design method will be obtained to synthesize PID controllers achieving a guaranteed cost such that the NCSs can be stabilized for all admissible uncertainties and time-delays. Finally, numerical examples are given to illustrate the effectiveness of the proposed method.

K e yw ords: PID controller, output feedback, Networked Control Systems (NCSs), polytopic system, parameterdependent quadratic stability, time-delay system
\end{abstract}

\section{INTRODUCTION}

Feedback control systems, wherein the loops are closed through real-time networks, are called Networked Control Systems (NCSs) [11,121416]. Advantages of using NCSs in the control area include simplicity, cost-effectiveness, ease of system diagnosis and maintenance, increased system agility and testability. However, integration of communication real-time networks into feedback control loops inevitably leads to some problems. As a result, it leads to a network-induced delay in the networked control closedloop system. The existence of such a kind of delay in a network-based control loop can induce instability or poor performance of control systems [8].

In the recent years, the stability analysis and controller synthesis for systems with time-delay are important in theory and practice [13]. In the time domain, there are two approaches to controller design and studying of stability of closed-loop systems: the Razumikhin theorem and the Lyapunov-Krasovskii functional (LKF) approach. It is well know that the LKF approach can provide less conservative results than the Razumikhin theorem [4613] and references therein. The existing criteria for asymptotic stability of the time-delay system can be classified into categories: delay-independent criteria and delay-dependent ones. It is also know that the delay-dependent criteria make use of information on the length of delays, they are less conservative than the delay-independent ones, even if the time delays are very small. On the other hand, a wide class of uncertainty types studied in the system and control literature fall into the polytopic perturbations. For the time-delay system with polytopic-type uncertainties, the parameterdependent stability condition is of lower conservativeness than the quadratic stability condition. Recently, freeweighting matrices method or slack-variable method and cross term bounding method were developed to obtain a less conservative condition [7 10] and reference therein.
The guaranteed cost control approach has been extended to the uncertain time-delay systems, for the state feedback case, see $[9,15,17]$ and for output feedback [5]. In the paper the authors consider the full order strictly proper dynamic output feedback controller. However, it seems that there is no previous result on the delaydependent guaranteed cost control via PID output feedback.

Motivated by the above observation, in this article the parameter-dependent, delay-dependent design method will be studied to design a robust output feedback PID controller achieving a guaranteed cost such that the NCSs can be stabilized for all admissible polytopic-type uncertainties and time-delays. The sufficient condition for the existence of a guaranteed cost output feedback controller is established in terms of matrix inequalities.

This paper is organized as follows. Section 2 gives the problem formulation. Section 3 explains the main results of the paper. In section 4, numerical examples are presented to show the effectiveness of the proposed method. Notation: Throughout this paper, for real matrix $M$, the notation $M \geq 0$ (or $M>0$ ) means that matrix $M$ is symmetric and positive semi-definite (or positive definite); "* denotes a block that is readily inferred by symmetry. Matrices, if not explicitly stated, are assumed to have compatible dimensions.

\section{PRELIMINARIES AND PROBLEM FORMULATION}

Consider the following linear time-delay system described

$$
\begin{aligned}
\dot{x}(t) & =A(\xi) x(t)+A_{d}(\xi) x(t-\tau)+B(\xi) u(t), \\
y t) & =C x(t), \\
x(t) & =\varphi(t), \quad t \in\left[-\tau_{M}, 0\right]
\end{aligned}
$$

* Slovak University of Technology, Faculty of Electrical Engineering and Information Technology, Ilkovičova 3 , 812 19 Bratislava, Slovakia; (thuan.quang,vojtech.vesely)@stuba.sk 
where $x(t) \in R^{n}$ is the state vector, $u(t) \in R^{m}$ is the control input, $y(t) \in R^{l}$ is the controlled output (measured output). Matrices $A(\xi), A_{d}(\xi), B(\xi) \in S$ belong to the convex hull, and $S$ is a polytope with $N$ vertices $S_{1}, S_{2}, \ldots, S_{N}$ which can formally be defined as

$$
S:=\left\{\begin{array}{c}
A(\xi), A_{d}(\xi) \in R^{n \times n}, B(\xi) \in R^{n \times m} \\
A(\xi)=\sum_{i=1}^{N} \xi_{i} A_{i}, A_{d}(\xi)=\sum_{i=1}^{N} \xi_{i} A_{d i}, \\
B(\xi)=\sum_{i=1}^{N} \xi_{i} B_{i}, \sum_{i=1}^{N} \xi_{i}=1, \xi_{i} \geq 0
\end{array}\right\}
$$

where $A_{i}, A_{d i}, B_{i}$ are constant matrices with appropriate dimensions and $\xi_{i}$ is time-invariant uncertainty, $\tau_{M}$ is the upper bound of time delay and $\varphi(t)$ is a continuously differentiable initial function. Note that $S$ is a convex and bounded domain. We assume that a real-time communication network is integrated into feedback control loops of system (1), and the network induced delay in NCS is given by $0<\tau \leq \tau_{M}$ and $\dot{\tau} \leq \mu \leq 1$.

For system (1), we consider the following PID control algorithm

$$
u(t)=K_{p} y(t-\tau)+K_{I} \int_{0}^{t} y(t-\tau) \mathrm{d} t+K_{D} \frac{\mathrm{d}}{\mathrm{d} t} y(t-\tau) .
$$

Consider $z(t)=\int y(t-\tau) \mathrm{d} t, \frac{\mathrm{d}}{\mathrm{d} t} y(t-\tau)=C_{d} \dot{x}(t-$ $\tau)$, where $C_{d}$ is an output matrix for derivative output feedback, and then by using the Newton-Leibniz formulas

$$
x(t-\tau)=x(t)-\int_{t-\tau}^{t} \dot{x}(s) \mathrm{d} s,
$$

and

$$
\dot{x}(t-\tau)=\dot{x}(t)-\int_{t-\tau}^{t} \ddot{x}(s) \mathrm{d} s,
$$

the PID control algorithm (3) can be written as

$$
\begin{aligned}
u(t)=F C_{n} X(t)+F_{D} C_{D} \dot{X}(t) & -F_{p} C_{p} \int_{t-\tau}^{t} \dot{X}(s) \mathrm{d} s \\
& -F_{d} C_{d} \int_{t-\tau}^{t} \ddot{X}(s) \mathrm{d} s,
\end{aligned}
$$

where

$$
\begin{gathered}
X(t)=\left[x^{\top}(t), z^{\top}(t)\right]^{\top}, \\
F=\left[\begin{array}{ll}
K_{P} & K_{I}
\end{array}\right], F_{P}=\left[\begin{array}{ll}
K_{P} & 0
\end{array}\right], F_{D}=\left[\begin{array}{ll}
K_{D} & 0
\end{array}\right], \\
C_{n}=\left[\begin{array}{ll}
C & 0 \\
0 & I
\end{array}\right], C_{P}=\left[\begin{array}{ll}
C & 0 \\
0 & 0
\end{array}\right], C_{D}=\left[\begin{array}{cc}
C_{d} & 0 \\
0 & 0
\end{array}\right] .
\end{gathered}
$$

Consider $\dot{z}(t)=C_{i} x(t-\tau)=C_{i} x(t)-C_{i} \int_{t-\tau}^{t} \dot{x}(s) \mathrm{d} s$, where $C_{i}$ is an output matrix for integral output feedback , the system (1) can be expanded in the following form

$$
\dot{X}(\xi)=A_{n}(\xi) X(t)+B_{n}(\xi) u(t)-A_{d n}(\xi) \int_{t-\tau}^{t} \dot{X}(s) \mathrm{d} s
$$

where

$$
\begin{aligned}
A_{n}(\xi) & =\sum_{i=1}^{N} \xi_{i} A_{n i}, & A_{n i} & =\left[\begin{array}{cc}
A_{i}+A_{d i} & 0 \\
C_{i} & 0
\end{array}\right], \\
B_{n}(\xi) & =\sum_{i=1}^{N} \xi_{i} B_{n i}, & B_{n i} & =\left[\begin{array}{c}
B_{i} \\
0
\end{array}\right] \\
A_{d n}(\xi) & =\sum_{i=1}^{N} \xi_{i} A_{d n i}, & A_{d n i} & =\left[\begin{array}{cc}
A_{d i} & 0 \\
C_{i} & 0
\end{array}\right] .
\end{aligned}
$$

Applying the PID control algorithm (4) to system (5) will result in the closed-loop system

$$
\begin{aligned}
M_{d}(\xi) \dot{X}(t)+A_{c}(\xi) X(t) & +A_{d c}(\xi) \int_{t-\tau}^{t} \dot{X}(s) \mathrm{d} s \\
& +A_{d d}(\xi) \int_{t-\tau}^{t} \ddot{X}(s) \mathrm{d} s=0,
\end{aligned}
$$

where

$$
\begin{aligned}
M_{d}(\xi) & =\sum_{i=1}^{N} \xi_{i} M_{d i}, & M_{d i} & =I-B_{n i} F_{D} C_{D} \\
A_{c}(\xi) & =\sum_{i=1}^{N} \xi_{i} A_{c i}, & A_{c i} & =-\left(A_{n i}+B_{n i} F C_{n}\right), \\
A_{d c}(\xi) & =\sum_{i=1}^{N} \xi_{i} A_{d c i}, & A_{d c i} & =A_{d n i}+B_{n i} F_{P} C_{P} \\
A_{d d}(\xi) & =\sum_{i=1}^{N} \xi_{i} A_{d d i}, & A_{d d i} & =B_{n i} F_{D} C_{D}
\end{aligned}
$$

Given positive definite symmetric matrices $Q, R$ and $S$, we will consider the cost function

$$
J=\int_{0}^{\infty} J(t) \mathrm{d} t
$$

where

$J(t)=X^{\top}(t) Q X(t)+u^{\top}(t) R u(t)+\dot{X}^{\top}(t-\tau) S \dot{X}(t-\tau)$. Consider $\eta(t)=$

$\left[\dot{X}^{\top}(t) \quad X^{\top}(t) \int_{t-\tau}^{t} \dot{X}^{\top}(s) \mathrm{d} s \int_{t-\tau_{M}}^{t-\tau} \dot{X}^{\top}(s) \mathrm{d} s \int_{t-\tau}^{t} \ddot{X}^{\top}(s) \mathrm{d} s\right]^{\top}$ and by substituting $u(t)$ from $(4)$ to $u^{\top}(t) R u(t)$ we obtain

$$
u^{\top}(t) R u(t)=\eta^{\top} K^{\top} R K \eta(t),
$$

where $K=\left[\begin{array}{lllll}F_{D} C_{D} & F C_{n} & -F_{p} C_{p} & 0 & -F_{D} C_{D}\end{array}\right]$. Using $\eta(t), J(t)$ can be rewritten as follows

$$
J(t)=\eta^{\top}(t) M_{Q}(\xi) \eta(t),
$$

where

$$
\begin{aligned}
& M_{Q}(\xi)=\left[\begin{array}{cc}
C_{D}^{\top} F_{D}^{\top} R F_{D} C_{D}+S & C_{D}^{\top} F_{D}^{\top} R F C_{n} \\
* & C_{n}^{\top} F^{\top} R F C_{n}+Q \\
* & * \\
* & * \\
* & *
\end{array}\right. \\
& -C_{D}^{\top} F_{D}^{\top} R F_{P} C_{P} \quad 0 \quad-C_{D}^{\top} F_{D}^{\top} R F_{D} C_{D}-S \\
& -C_{n}^{\top} F^{\top} R F_{P} C_{P} \quad 0 \quad-C^{\top} F^{\top} R F_{D} C_{D} \\
& C_{P}^{\top} F_{P}^{\top} R F_{P} C_{P} \quad 0 \quad C_{P}^{\top} F_{P}^{\top} R F_{D} C_{D} \\
& \begin{array}{ccc}
* & 0 & 0 \\
* & * & C_{D}^{\top} F_{D}^{\top} R F_{D} C_{D}+S
\end{array}
\end{aligned}
$$


Associated with the cost, the guaranteed cost controller is defined as follows:

Definition 1. Consider the uncertain system (1). If there exists a controller of form (3) and a positive scalar $J_{0}$ such that for all uncertainties (2) the closed-loop system (6) is asymptotically stable and closed-loop value of the cost function (7) satisfies $J \leq J_{0}$ then $J_{0}$ is said to be a guaranteed cost and the controller (2) is said to be a guaranteed cost controller.

Finally we introduce the well known results from LQ theory.

LEMma 1. Consider the continuous-time delay system (5) with control algorithm (3). The control algorithm (3) is the guaranteed cost control for system (5) if and only if there exists $\operatorname{LKF} V(\xi, t)$ such that the following condition holds

$$
\frac{d}{d t} V(\xi, t)+J(t) \leq 0 .
$$

The objective of this paper is to develop a procedure to design a robust PID controller of form (4) which ensures parameter-dependent the closed-loop system stability and guaranteed cost.

\section{MAIN RESULTS}

The following theorem provides robust parameterdependent quadratic stability and robust performance results for the closed-loop system (6).

THEOREM 1. Consider the uncertain linear time-delay system (1) with network-induced delay $\tau$ satisfying $0 \leq$ $\tau \leq \tau_{M}, \dot{\tau} \leq \mu \leq 1$ and the cost function (7). If there exists a PID controller of form (3), scalar $J_{0}$, and matrices $P_{i}>0, G_{i}>0, G_{1 i}>0, G_{2 i}>0, G_{3 i}>0$ $(i=1, \ldots, N), N_{1}, N_{2}, N_{3}, N_{4}$, and $N_{5}$ that satisfy the following matrix inequality

$$
W_{i}=\left[\begin{array}{ccccc}
w_{i}^{11} & w_{i}^{12} & w_{i}^{13} & w_{i}^{14} & w_{i}^{15} \\
* & w_{i}^{22} & w_{i}^{23} & w_{i}^{24} & w_{i}^{15} \\
* & * & w_{i}^{33} & w_{i}^{34} & w_{i}^{35} \\
* & * & * & w_{i}^{44} & w_{i}^{45} \\
* & * & * & * & w_{i}^{55}
\end{array}\right] \leq 0
$$

where

$$
\begin{aligned}
w_{i}^{11} & =N_{1} M_{d i}+M_{d i}^{\top} N_{1}^{\top}+\tau_{M} G_{1 i}+\mu G_{3 i}+ \\
& C_{D}^{\top} F_{D}^{\top} R F_{D} C_{D}+S \\
w_{i}^{12}= & N_{1} A_{c i}+M_{d i}^{\top} N_{2}^{\top}+P_{i}+C_{D}^{\top} F_{D}^{\top} R F C_{n}, \\
w_{i}^{13}= & N_{1} A_{d c i}+M_{d i}^{\top} N_{3}^{\top}-C_{D}^{\top} F_{D}^{\top} R F_{P} C_{P}, \\
w_{i}^{14}= & M_{d i}^{\top} N_{4}^{\top} \\
w_{i}^{15}= & N_{1} A_{d d i}+M_{d i}^{\top} N_{5}^{\top}+(1-\mu) \mu G_{3 i} \\
& -C_{D}^{\top} F_{D}^{\top} R F_{D} C_{D}-S, \\
w_{i}^{22}= & N_{2} A_{c i}+A_{c i}^{\top} N_{2}^{\top}+\mu G_{i}+C_{n}^{\top} F^{\top} R F+Q,
\end{aligned}
$$

$$
\begin{aligned}
& w_{i}^{23}=N_{2} A_{d c i}+A_{c i}^{\top} N_{3}^{\top}+(1-\mu) G_{i}+G_{2 i} \\
&-C_{n}^{\top} F^{\top} R F_{P} C_{P}, \\
& w_{i}^{24}=A_{c i}^{\top} N_{4}^{\top}+G_{2 i}, \\
& w_{i}^{25}=N_{2} A_{d d i}+A_{c i}^{\top} N_{5}^{\top}-C_{n}^{\top} N^{\top} R F_{D} C_{D}, \\
& w_{i}^{33}=N_{3} A_{d c i}+A_{d c i}^{\top} N_{3}^{\top}-(1-\mu) G_{i}-\frac{1}{\tau_{M}} G_{1 i}-G_{2 i}+ \\
& C_{P}^{\top} F_{P}^{\top} R F_{P} C_{P}, \\
& w_{i}^{34}=A_{d c i}^{\top} N_{4}^{\top}-G_{2 i}, \\
& w_{i}^{35}=N_{3} A_{d d i}+A_{d c i}^{\top} N_{5}^{\top}+C_{P}^{\top} F_{P}^{\top} R F_{D} C_{D}, \\
& w_{i}^{44}=-G_{2 i}-\frac{1}{\tau_{M}} G_{1 i}, \\
& w_{i}^{45}=N_{4} A_{d d i}, \\
& w_{i}^{55}=N_{5} A_{d d i}+A_{d d i}^{\top} N_{5}^{\top}-(1-\mu) G_{3 i}+ \\
& C_{D}^{\top} F_{D}^{\top} R F_{D} C_{D}+S .
\end{aligned}
$$

then the uncertain system (1) with controller (3) is parameter-dependent quadratically stable and the cost function (7) satisfies the following bound

$$
J \leq J_{0}=\sqrt{\lambda_{M P}^{2}+\lambda_{M G}^{2}+\lambda_{M G 1}^{2}+\lambda_{M G 2}^{2}+\lambda_{M G 3}^{2}} * J_{M}
$$

where

$$
\begin{gathered}
\lambda_{M P}=\operatorname{Max}_{i=1 \ldots N}\left(\operatorname{Max} \operatorname{Eigenvalue}\left(P_{i}\right)\right), \\
\lambda_{G_{i}}=\operatorname{Max}_{i=1 \ldots N}\left(\operatorname{Max} \operatorname{Eigenvalue}\left(G_{i}\right)\right), \\
\lambda_{M G 1}=\underset{i=1 \ldots N}{\operatorname{Max}}\left(\operatorname{Max} \operatorname{Eigenvalue}\left(G_{1 i}\right)\right), \\
\lambda_{M G 2}=\operatorname{Max}_{i=1 \ldots N}\left(\operatorname{Max} \operatorname{Eigenvalue}\left(G_{2 i}\right)\right), \\
\lambda_{M G 3}=\operatorname{Max}_{i=1 \ldots N}\left(\operatorname{Max} \operatorname{Eigenvalue}\left(G_{3 i}\right)\right), \\
J_{M}=\left(\left\|x_{0}\right\|^{4}+\left(\int_{-\tau}^{0}\|\varphi(s)\|^{2} d s\right)^{2}+\left(\int_{-\tau}^{0} d \theta \int_{\theta}^{0}\|\dot{\varphi}(s)\|^{2} d s\right)^{2}\right. \\
\left.+\left(\int_{-\tau}^{-\tau}\|\varphi(s)\|^{2} d s\right)^{2}+\left(\int_{-\tau}^{0}\|\dot{\varphi}(s)\|^{2} d s\right)^{2}\right)^{1 / 2} .
\end{gathered}
$$

P r o o f . Consider the Lyapunov-Krasovskii functional as follows

$$
\begin{aligned}
V(\xi, t) & =\sum_{i=1}^{5} V_{i}(\xi, t), \\
V_{1}(\xi, t) & =X^{\top}(t) P(\xi) X(t), \\
V_{2}(\xi, t) & =\int_{t-\tau}^{t} X^{\top}(s) G(\xi) X(s) \mathrm{d} s, \\
V_{3}(\xi, t) & =\int_{-\tau}^{0} \mathrm{~d} \theta \int_{t+\theta}^{t} \dot{X}^{\top}(s) G_{1}(\xi) \dot{X}(s) \mathrm{d} s, \\
V_{4}(\xi, t) & =\int_{t-\tau_{M}}^{t} X^{\top}(s) G_{2}(\xi) X(s) \mathrm{d} s,
\end{aligned}
$$




$$
V_{5}(\xi, t)=\int_{t-\tau}^{t} \dot{X}^{\top}(s) G_{3}(\xi) \dot{X}(s) \mathrm{d} s .
$$

Differentiating $V(\xi, t)$ with respect to $t$ and using the Newton-Leibniz formula $x(t-\tau)=x(t)-\int_{t-\tau}^{t} \dot{x}(s) \mathrm{d} s$ we obtain

$$
\begin{aligned}
& \dot{V}_{1}(\xi, t)=z X^{\top}(t) P(\xi) \dot{X}(t), \\
& \dot{V}_{2}(\xi, t) \leq \eta_{1}^{\top}(t)\left[\begin{array}{cc}
\mu G(\xi) & (1-\mu) G(\xi) \\
* & -(1-\mu) G(\xi)
\end{array}\right] \eta_{1}(t), \\
& \eta_{1}^{\top}(t)=\left[\begin{array}{ll}
X^{\top}(t) & \int_{t-\tau}^{t} \dot{X}^{\top}(s) \mathrm{d} s
\end{array}\right], \\
& \dot{V}_{3} \leq \tau_{M} \dot{X}^{\top}(t) G_{1}(\xi) \dot{X}(t) \\
& -\frac{1}{\tau_{M}} \int_{t-\tau}^{t} \dot{X}^{\top}(s) \mathrm{d} s G_{1}(\xi) \int_{t-\tau}^{t} \dot{X}(s) \mathrm{d} s, \\
& \dot{V}_{4}(\xi, t) \leq \eta_{2}^{\top}(t)\left[\begin{array}{ccc}
0 & G_{2}(\xi) & G_{2}(x i) \\
* & -G_{2}(x i) & -G_{2}(x i) \\
* & * & -G_{2}(x i)
\end{array}\right] \eta_{2}(t), \\
& \eta_{2}^{\top}(t)=\left[\begin{array}{lll}
X^{\top}(t) & \int_{t-\tau}^{t} \dot{X}^{\top}(s) \mathrm{d} s & \int_{t-\tau_{M}}^{t-\tau} \dot{X}^{\top}(s) \mathrm{d} s
\end{array}\right], \\
& \dot{V}_{5}(\xi, t) \leq \eta_{3}^{\top}(t)\left[\begin{array}{cc}
\mu G_{3}(\xi) & (1-\mu) G_{3}(\xi) \\
* & -(1-\mu) G_{3}(\xi)
\end{array}\right] \eta_{3}(t), \\
& \eta_{3}^{\top}=\left[\begin{array}{ll}
X^{\top} & \int_{t-\tau} \ddot{X}^{\top}(s) \mathrm{d} s
\end{array}\right] .
\end{aligned}
$$

Applying the free-weighting matrices technique, equation (8) is represented in the following equivalent form

$$
\begin{gathered}
\alpha(t)=2 \eta^{\top}(t)\left[\begin{array}{lllll}
N_{1}^{\top} & N_{2}^{\top} & N_{3}^{\top} & N_{4}^{\top} & N_{5}^{\top}
\end{array}\right]^{\top} \\
{\left[\begin{array}{lllll}
M_{d}(\xi) & A_{c}(\xi) & A_{d c}(\xi) & 0 & A_{d d}(\xi)
\end{array}\right] \eta(t)=0 .}
\end{gathered}
$$

After manipulation of the above equation, we obtain

$$
\alpha(t)=\eta^{\top}(t) M_{\alpha}(\xi) \eta(t)=0
$$

where

$$
\begin{aligned}
& M_{\alpha}(\xi)=\left[\begin{array}{cc}
N_{1} M_{d}(\xi)+M_{d}^{\top}(\xi) N_{1}^{\top} & N_{1} A_{c}(\xi)+M_{d}^{\top}(\xi) N_{2}^{\top} \\
* & N_{2} A_{c}(\xi)+A_{c}^{\top}(\xi) N_{2}^{\top} \\
* & * \\
* & * \\
* & *
\end{array}\right. \\
& \left.\begin{array}{ccc}
N_{1} A_{d c}(\xi)+M_{d}^{\top}(\xi) N_{3}^{\top} & M_{d}^{\top}(\xi) N_{4}^{\top} & N_{1} A_{d d}(\xi)+M_{d}^{\top}(\xi) N_{5}^{\top} \\
N_{2} A_{d c}(\xi)+A_{c}^{\top}(\xi) N_{3}^{\top} & A_{c}^{\top}(\xi) N_{4}^{\top} & N_{2} A_{d d}(\xi)+A_{c}^{\top}(\xi) N_{5}^{\top} \\
N_{3} A_{d c}(\xi)+A_{d c}^{\top}(\xi) N_{3}^{\top} & A_{d c}(\xi) N_{4}^{\top} & N_{3} A_{d c}(\xi)+A_{d c}^{\top}(\xi) N_{5}^{\top} \\
* & 0 & N_{4} A_{d d}(\xi) \\
* & * & N_{5} A_{d d}(\xi)+A_{d d}^{\top}(\xi) N_{5}^{\top}
\end{array}\right] .
\end{aligned}
$$

Because of $\alpha(t)=0$, thus

$$
\begin{aligned}
\dot{V}(\xi, t)=\sum_{i=1}^{5} \dot{V}_{i}(\xi, t)+\alpha(t) \leq \eta^{\top}(t) & {\left[M_{\alpha}(\xi)\right.} \\
& \left.+M_{V}(\xi)\right] \eta^{\top}(t),
\end{aligned}
$$

where

$$
M_{V}(\xi)=\left[\begin{array}{ccc}
\tau_{M} G_{1}(\xi)+\mu G_{3}(\xi) & P(\xi) & \\
* & \mu G(\xi) & \\
* & * & \\
* & * & \\
* & * & (1-\mu) G_{3}(\xi) \\
0 & 0 & 0 \\
(1-\mu) G(\xi) & G_{2}(\xi) & 0 \\
-(1-m u) G(\xi)-G_{2}(\xi) & -G_{2}(\xi) & 0 \\
-\frac{1}{\tau_{M}} G_{1}(\xi) & -G_{2}(\xi)-\frac{1}{\tau_{M}} G_{1}(\xi) & -(1-\mu) G_{3}(\xi)
\end{array}\right]
$$

Due to Lemma 1, the closed-loop system (6) is robustly asymptotically stable and give an upper bound (a guaranteed cost) for the cost function (7) if

$$
\dot{V}(\xi, t)+J(t) \leq \eta^{\top}(t) W(\xi) \eta^{\top}(t) \leq 0 \Longleftrightarrow W(\xi) \leq 0,
$$

where $W(\xi)=\sum_{i=1}^{N} \xi_{i} W_{i}=M_{\alpha}(\xi)+M_{V}(\xi)+M_{Q}(\xi)$. If for each $W_{i} \leq 0, I=1, \ldots, N$, then $W(\xi)=$ $\sum_{i-1}^{N} \xi_{i} W_{i} \leq 0$. Therefore, $\dot{V}(\xi, t) \leq-J(t) \leq 0(J(t)>$ $0)$, or $J(t) \leq-\dot{V}(\xi, t)$. By integrating $J(t) \leq-\dot{V}(\xi, t)$ we obtain

$J \leq-\int_{0}^{\infty} \dot{V}(\xi, t)=V_{0}=X_{0}^{\top} P(\xi) X_{0}+$

$$
\begin{array}{r}
\int_{-\tau}^{0} X^{\top}(s) G(\xi) X(s) \mathrm{d} s+\int_{-\tau}^{0} \mathrm{~d} \theta \int_{\theta}^{0} \dot{X}^{\top}(s) G_{1}(\xi) \dot{X}(\xi) \mathrm{d} s \\
+\int_{-\tau_{M}}^{0} X^{\top} G_{2}(\xi) X(s) \mathrm{d} s+\int_{-\tau}^{0} \dot{X}^{\top}(s) G_{3}(\xi) \dot{X}(s) \mathrm{d} s
\end{array}
$$

Because of $X(t)=\left[\varphi^{\top}(t) \quad 0\right], \forall t \in\left[-\tau_{M}, 0\right]$

$$
\begin{aligned}
V_{0} \leq \lambda_{M P}\left\|x_{0}\right\|^{2}+\lambda_{M G} \int_{-\tau}^{0}\|\varphi(s)\|^{2} \mathrm{~d} s & \\
+\lambda_{M G 1} \int_{-\tau}^{0} \mathrm{~d} \theta \int_{\theta}^{0}\|\dot{\varphi}(s)\|^{2} \mathrm{~d} s & +\lambda_{M G 2} \int_{-\tau_{M}}^{0}\|\varphi(s)\|^{2} \mathrm{~d} s \\
& +\lambda_{M G 3} \int_{-\tau}^{0}\|\dot{\varphi}(s)\|^{2} \mathrm{~d} s .
\end{aligned}
$$

It is known that for two arbitrary vectors $\boldsymbol{X}, \boldsymbol{Y}$, the following inequality hold

$$
|\boldsymbol{X Y}| \leq\|\boldsymbol{X}\|\|\boldsymbol{Y}\| .
$$

Consider $\vec{X}=\left[\begin{array}{lllll}\lambda_{M P} & \lambda_{M G} & \lambda_{M G 1} & \lambda_{M G 2} & \lambda_{M G 3}\end{array}\right]^{\top}$, $\boldsymbol{Y}=\left[\left\|x_{\mathrm{o}}\right\|^{2} \int_{-\tau}^{0}\|\varphi(s)\|^{2} \mathrm{~d} s\right.$

$$
\left.\int_{-\tau}^{0} \mathrm{~d} \theta \int_{\theta}^{0}\|\varphi(s)\|^{2} \mathrm{~d} s \int_{-\tau_{M}}^{0}\|\varphi(s)\|^{2} \mathrm{~d} s \int_{-\tau}^{0}\|\varphi(s)\|^{2} \mathrm{~d} s\right]^{\top} .
$$

Applying inequality (16) to the above equation the upper bound cost function (7) $J_{0}$ is obtained as (11). The theorem 1 is proved. 


\section{EXAMPLES}

In this section we present the results of numerical calculations of two examples to design a robust output feedback PID controller with guaranteed cost for NCSs with time-delay. The design procedure is based on BMI inequalities (10).

Example 1 has been borrowed from [2] to demonstrate the use of algorithm (10) for the problem of robustly stabilizing, with a guaranteed cost, vertical take-off and landing of a helicopter. The system is controlled through NCS with a time-varying time- delay $0<\tau \leq \tau_{M}=$ $200 \mathrm{~ms}, \dot{\tau} \leq \mu \leq 0.99$. Let uncertain matrices $A, B, C$, $A_{d}$ be defined as

$$
\begin{gathered}
A=\left[\begin{array}{cccc}
-0.036 & 0.0270 & 0.0188 & -0.4555 \\
0.0482 & -1.010 & 0.0024 & -4.0208 \\
0.1002 & q_{1}(t) & -0.707 & q_{2}(t) \\
0 & 0 & 1 & 0
\end{array}\right], \\
B=\left[\begin{array}{cc}
4.422 & 0.1761 \\
q_{3}(t) & -7,59222 \\
-5.52 & 4.49 \\
0 & 0
\end{array}\right], C^{\top}=\left[\begin{array}{l}
0 \\
1 \\
0 \\
0
\end{array}\right], A_{d}=0
\end{gathered}
$$

with parameters bounds for all time $-0.6319 \leq q_{1}(t) \leq$ $1.3691,1.22 \leq q_{2}(t) \leq 1.420,2.7446 \leq q_{3}(t) \leq 4.3446$. The above model has been recalculated to the form (1). The respective eight vertices are calculated. Note that matrix $A$ is unstable with max real eigenvalue $(A)=$ 1.2675. The results of calculation for the case $r=1$, $q=0.1, s=0.001, r_{0}=10$ are as follows

$$
F=\left[\begin{array}{ll}
K_{P} & K_{I}
\end{array}\right]=\left[\begin{array}{cc}
-0.2788 & 0.0927 \\
0.5857 & 0.4086
\end{array}\right], K_{D} \cong 0 .
$$

The maxreal eigenvalue $($ Close-loop $)=-0.072209$ and guaranteed cost $J_{0}=17.9128 J_{M}$, where $\lambda_{M P}=\lambda_{M G}=$ $\lambda_{M G 2}=8.9794, \lambda_{M G 1}=8.8841, \lambda_{M G 3}=0.2319$.

EXAMPLE 2. We consider the linear model of two cooperating DC motors. The problem is to design two PI controllers for a laboratory MIMO system which guarantee robust stability with a guaranteed cost. The system is controlled through a NCS with time-varying time-delay $0<\tau \leq \tau_{M}=100 \mathrm{~ms}, \dot{\tau} \leq \mu=0.2$. The system model is given with a time invariant matrix affine type uncertain structure, where

$$
\begin{aligned}
A_{0}= & {\left[\begin{array}{cccccccc}
0 & -.2148 & 0 & 0 & 0 & 0 & 0 & 0 \\
1 & -1.014 & 0 & 0 & 0 & 0 & 0 & 0 \\
0 & 0 & 0 & -.2605 & 0 & 0 & 0 & 0 \\
0 & 0 & 1 & -.9107 & 0 & 0 & 0 & 0 \\
0 & 0 & 0 & 0 & 0 & -.1639 & 0 & 0 \\
0 & 0 & 0 & 0 & 1 & -.8137 & 0 & 0 \\
0 & 0 & 0 & 0 & 0 & 0 & 0 & -.2279 \\
0 & 0 & 0 & 0 & 0 & 0 & 1 & -.8251
\end{array}\right], } \\
B_{0}= & {\left[\begin{array}{cc}
.3148 & 0 \\
.0478 & 0 \\
0 & -.1028 \\
0 & -.0091 \\
-.0841 & 0 \\
-.0287 & 0 \\
0 & .3676 \\
0 & .2448
\end{array}\right], B_{1}=\left[\begin{array}{ccc}
.0625 & 0 \\
.0798 & 0 \\
0 & -.0462 \\
0 & -.0449 \\
.0016 & 0 \\
.0072 & 0 \\
0 & .077 \\
0 & .003
\end{array}\right] }
\end{aligned}
$$

$$
\begin{aligned}
& A_{1}=\left[\begin{array}{cccccccc}
0 & -.025 & 0 & 0 & 0 & 0 & 0 & 0 \\
0 & -.1395 & 0 & 0 & 0 & 0 & 0 & 0 \\
0 & 0 & 0 & -.0938 & 0 & 0 & 0 & 0 \\
0 & 0 & 0 & -.2911 & 0 & 0 & 0 & 0 \\
0 & 0 & 0 & 0 & 0 & .0188 & 0 & 0 \\
0 & 0 & 0 & 0 & 0 & .0208 & 0 & 0 \\
0 & 0 & 0 & 0 & 0 & 0 & 0 & -.0333 \\
0 & 0 & 0 & 0 & 0 & 0 & 0 & -.1173
\end{array}\right], \\
& A_{2}=\left[\begin{array}{cccccccc}
0 & .0125 & 0 & 0 & 0 & 0 & 0 & 0 \\
0 & .0594 & 0 & 0 & 0 & 0 & 0 & 0 \\
0 & 0 & 0 & .0116 & 0 & 0 & 0 & 0 \\
0 & 0 & 0 & .0308 & 0 & 0 & 0 & 0 \\
0 & 0 & 0 & 0 & 0 & -.0188 & 0 & 0 \\
0 & 0 & 0 & 0 & 0 & -.0156 & 0 & 0 \\
0 & 0 & 0 & 0 & 0 & 0 & 0 & .0208 \\
0 & 0 & 0 & 0 & 0 & 0 & 0 & -.0333
\end{array}\right], \\
& B_{2}=\left[\begin{array}{cc}
.0094 & 0 \\
.0151 & 0 \\
0 & .0019 \\
0 & -.003 \\
-.0121 & 0 \\
-.03 & 0 \\
0 & -.064 \\
0 & .0189
\end{array}\right] \\
& C=\left[\begin{array}{llllllll}
0 & 1 & 0 & 1 & 0 & 0 & 0 & 0 \\
0 & 0 & 0 & 0 & 0 & 1 & 0 & 1
\end{array}\right], A_{d}=0 \text {. }
\end{aligned}
$$

The above model has been recalculated to the form (1). The respective four vertices are calculated. The results of calculation for the case $r=1, q=0.1, s=0.001$, $r_{0}=20$ are as follows

$$
F=\left[\begin{array}{ll}
K_{P} & K_{I}
\end{array}\right]=\left[\begin{array}{cccc}
-1.8916 & 0.1128 & 0.2026 & -0.6964 \\
-0.4310 & -1.8916 & 0.8782 & -1.4985
\end{array}\right]
$$

The max realeigenvalue $($ Close-loop $)=-0.17726$. And guaranteed cost $J_{0}=37.7659 J_{M}$ where $\lambda_{M P}=18.916$, $\lambda_{M G}=15.0011, \lambda_{M G 2}=18.916, \lambda_{M G 1}=15.5723$, $\lambda_{M G 3}=15.5918$.

\section{CONCLUSION}

The guaranteed cost control problem is studied in this paper for a class of linear time-delay uncertain polytopic systems and a given quadratic cost function with three terms (QRS). On the basis of the Lyapunov-Krasovskii functional, new sufficient parameter-dependent quadratic stability conditions are given for the output feedback PID controller proposed design procedure in terms of bilinear matrix inequality.

The examples show the effectiveness of the proposed method.

\section{Acknowledgment}

The work has been supported by Grant N1/0544/09 of the Slovak Scientific Grant Agency. 


\section{REFERENCES}

[1] BASIN, M.-PEREZ, J.-MARTINEZ-ZUNIGA : Optimal Filtering for Nonlinear Polynomial Systems over Linear Observation with Delay, Int. J. Innovative Computing, Information and Control 2 No. 4 (2006), 863-874.

[2] BENTON, R. E. jr.-SMITH, D. : A Non Iterative LMI Based Algorithm for Robust Static Output Feedback Stabilization, Int. J. Control 72 No. 14 (1999), 1322-1330.

[3] BOUKAZ, E. K.-Al-MUTHAIRI, N. F. : Delay-Dependent Stabilization of Singular Linear Systems with Delays, Int. J. Innovative Computing, Information and Control 2 No. 2 (2006), 283-291.

[4] CHARITONOV, V. L.-MELCHELOR-ARQUILAR, D. : On Delay-Dependent Stability Conditions, Systems and Control Letters 40 (2000), 71-76.

[5] CHEN, W. H.-GUAN, Z. H.-LU, X. : Delay-Dependent Output Feedback Guaranteed Cost for Uncertain Time-Delay Systems, Automatica 40 (2004), 1263-1268.

[6] FRIDMAN, E.-NICULESCU, S. J.: On Complete Lyapunov-Krasovskii Functional Techniques for Uncertain Systems with Fast Varying Delays, Int. J. of Robust and Nonlinear Control 18 (2008), 364-374.

[7] HE, Y.-WAN, Q. G.-XIE, L.-LIN, C.: Further Improvement of Free-Weighting Matrices Technique for Systems with Time-Varying Delay, IEEE Trans. Automat. Contr. 52 (2007), 293-298.

[8] JIANG, X.-HAN, Q. L. : New Stability Criteria for Linear Systems with Interval Time-Varying Delay, Automatica 44 (2008), 2680-2685.

[9] LEE, B.-GUYLEE, J. : Robust Stability and Stabilization of Linear Delayed Systems with Structured Uncertainty, Automatica 35 (1999), 1149-1154.

[10] MONDIE, S.-KHARITONOV, V.-SANTOS, O. : Complete LyapunovKrasovskii Functional with a Given Cross Term in the Time Derivative, Proceedings of $44^{\text {th }}$ Conference on Decision and Control, Sevilla, Spain, 2005, pp. 5060-5064.

[11] NILSON, J.: Real-Time Control System with Delays, PhD disertation, Department of Automatic Control, Lund Institute of Technology, Lund, Sweden, 1998.
[12] RAY, A.-HALEVI, Y.: Integrated Communication and Control Systems: Part II Design Considerations, ASME Journal of Dynamic Systems, Measurement and Control 110 (1988), $374-381$.

[13] RICHARD, J. P.: Time-Delay Systems: an Overview of some Recent Advances and Open Problem, Automatica 39 (2003), 1667-1694.

[14] WALSH, G. C.-YE, H.-BUSHNELL, L.: Stability Analysis of Networked Control Systems, Procedings of the American Control Conference, 1999, pp. 2876-2880.

[15] YU, L.-CHU, J.: An LMI Approach to Guaranteed Cost Control of Linear Uncertaint Time-Delay Systems, Automatica 35 (1999), 1155-1159.

16] ZHANG, W.-BRANICKY-PHILIPS, S. M. : Stability of Networked Control Systems, IEEE Control System Magazine 21 No. 1 (200), 84-99.

17] ZHANG, L.-El-KEBIR, B.-HAIDAR, A. : Delay-Range Depdendent Control Synthesis for Time-d.

Received 19. October 2009

Nguyen Quang Thuan born in 1982 in Hanoi-VietNam. Has been studying from 2003 to August 2007 for Bachelor of Science at Faculty of Electrical Engineering and Information Technology, Slovak University of Technology in Bratislava, Slovakia and from 2007 to 2009 for Master of Science at the same place, where he is from 2009 until now doing further studies to get his $\mathrm{PhD}$ title.

Vojtech Veselý (Prof, Ing, DrSc) was born in 1940. Since 1964 he has worked at the Department of Automatic Control Systems at the Faculty of Electrical Engineering and Information Technology, Slovak University of Technology in Bratislava, where he has supervised $19 \mathrm{PhD}$ students. Since 1986 he has been Full Professor. His research interests include the areas of power system control, decentralized control of large-scale systems, process control and optimization. He is author and coauthor of more than 250 scientific papers.

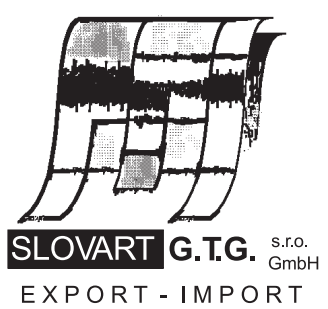

EXPORT - IMPORT
EXPORT - IMPORT

of periodicals and of non-periodically printed matters, books and CD-ROMs

Krupinská 4 PO BOX 152, 85299 Bratislava 5, Slovakia tel: ++421 263839 472-3, fax: ++421263839485 info@slovart-gtg.sk; http://www.slovart-gtg.sk

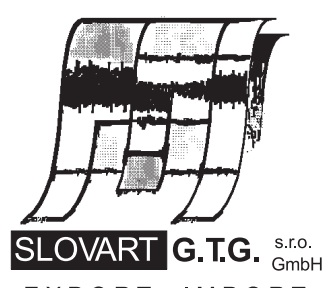

EXPORT - IMPORT 